\title{
Associação Entre a Densidade Radiológica da Placa à Tomografia de Coronárias com 64 Colunas de Detectores e a Composição da Placa ao Ultrassom Intravascular com Técnica de Histologia Virtual: Resultados de uma Comparação Pareada Prospectiva
}

\author{
João Luiz de Alencar Araripe Falcão', Swaminatha V. Gurudevan², Afonso Akio Shiozaki', \\ Otávio Rizzi Coelho Filho', Breno de Alencar Araripe Falcão ${ }^{1}$, Fernando José Ribeiro Sales ${ }^{1}$, \\ Eulógio Emílio Martinez Filho1, Expedito Eustáquio Ribeiro', Carlos Eduardo Rochitte', \\ Jagat Narula ${ }^{2}$, Pedro Alves Lemos Neto
}

\section{RESUMO}

Introdução: Pouco se conhece sobre a comparação entre métodos de avaliação da placa aterosclerótica coronária, como a tomografia computadorizada com múltiplos detectores (TCMD) e o ultrassom intravascular com técnica de histologia virtual (HV). Método: Foram analisados prospectivamente os vasos coronários principais de 21 pacientes à TCMD e à HV. Cada vaso analisado foi dividido em subsegmentos de $4 \mathrm{~mm}$ (unidades básicas para a análise). Os subsegmentos foram pareados conforme sua localização axial. Os limites da luz e do vaso foram traçados à HV e à TCMD com o auxílio de programas específicos. A contribuição porcentual de cada componente da placa à HV e a razão necrose:cálcio foram quantificadas. A placa à TCMD foi avaliada por sua densidade radiológica em unidades Hounsfield (UH). Resultados: Foram analisados 641 subsegmentos, obtidos de 70 vasos. A contribuição porcentual dos componentes à HV foi: fibroso $=64 \%$; fibrolipídico $=20 \%$; núcleo necrótico $=11 \%$; cálcio denso $=$ $5 \%$; e razão necrose:cálcio $=5,1$. A atenuação da placa à TCMD variou de $44 \mathrm{UH}$ a $440 \mathrm{UH}$. Para a análise, as placas foram divididas em três grupos de tamanhos semeIhantes, conforme a densidade da placa à TCMD (1ำ tercil: 44-128 UH, 2o tercil: 129-178 UH, e $3^{\circ}$ tercil: 178-440 UH).
ABSTRACT

Association Between Plaque Radiological Density by 64-Slice Multidetector Computed Tomography and Plaque Composition by Intravascular Ultrasound Virtual Histology: Results from a Prospective Matched Comparison

Background: Little is known about the comparative results of coronary plaque assessment by multidetector computed tomography (MDCT) against the novel intravascular ultrasound virtual histology (IVUS-VH). Method: Threevessel IVUS-VH and MDCT were prospectively obtained from 21 patients. Each vessel was divided into 4-mm subsegments, which were used as the basic unit for analysis. The 4-mm subsegments were matched by their axial location. Lumen and outer vessel boundaries for IVUS-VH and for MDCT were traced by specific automatic softwares. The percent contribution of each IVUS-VH plaque tissue type and the ratio of necrotic tissue to calcium were quantified. The MDCT plaque was evaluated through its average density in Hounsfield units (HU). Results: A total of 641 matched subsegments were computed from 70 vessels. Overall, the average IVUS-VH tissue composition was: fibrous $=64 \%$; fibrofatty $=20 \%$; necrotic tissue $=11 \%$;

Suporte Financeiro: FAPESP - Fundação de Amparo à Pesquisa do Estado de São Paulo/SOCESP - Sociedade de Cardiologia do Estado de São Paulo.

1 Instituto do Coração do Hospital das Clínicas da Faculdade de Medicina da Universidade de São Paulo - São Paulo, SP, Brasil. ${ }^{2}$ Cardiovascular Imaging Center for Education and Research (CVICER)

- University of California, Irvine (UCI) School of Medicine - Irvine, Estados Unidos.

Correspondência: João Luiz de Alencar Araripe Falcão. Rua Alves Guimarães, 408 - São Paulo, SP, Brasil - CEP 05410-000

E-mail: jlfalcao@uol.com.br

Recebido em: 5/8/2009 • Aceito em: 13/9/2009 
Falcão JLAA, et al. Associação Entre a Densidade Radiológica da Placa à Tomografia de Coronárias com 64 Colunas de Detectores e a Composição da Placa ao Ultrassom Intravascular com Técnica de Histologia Virtual: Resultados de uma Comparação Pareada Prospectiva. Rev Bras Cardiol Invas. 2009;17(3):327-34.

Houve correlação inversa entre a razão necrose/cálcio à HV e densidade da placa à TCMD (1ำ tercil: 4,1, $2^{\circ}$ tercil: 2,9, e $3^{\circ}$ tercil: 2,$\left.3 ; \mathrm{P}<0,01\right)$ e relação direta entre o porcentual de cálcio denso à $\mathrm{HV}$ e a densidade da placa à TCMD (1을 tercil: $1,5 \%$, 2 o tercil: $2,5 \%$, e $3^{\circ}$ tercil: $3,8 \%$; $P<0,01)$. Conclusão: A densidade da placa aterosclerótica avaliada pela TCMD em tomógrafo de 64 colunas de detectores associa-se significativamente com a composição da placa à HV.

DESCRITORES: Tomografia computadorizada espiral. Ultrasonografia de intervenção. Doença da artéria coronariana/ patologia. dense calcium $=5 \%$; and calcium $/$ necrosis ratio $=5.1$. MDCT average plaque attenuation ranged from 44 to $440 \mathrm{HU}$ and was divided into three groups of similar size $\left(1^{\text {st }}\right.$ tertile: 44-128 HU; $2^{\text {nd }}$ tertile: $129-178 \mathrm{HU}$; $3^{\text {rd }}$ tertile: $178-440 \mathrm{HU}$ ). There was an inverse correlation between IVUS-VH necrotic/ dense calcium ratio and MDCT plaque density ( $1^{\text {st }}$ tertile: 4.1, $2^{\text {nd }}$ tertile: $2.9,3^{\text {rd }}$ tertile: $\left.2.3 ; \mathrm{P}<0.01\right)$ and a direct relationship between percent IVUS-VH dense calcium and MDCT plaque density ( $1^{\text {st }}$ tertile: $1.5 \%, 2^{\text {nd }}$ tertile: $2.5 \%$, $3^{\text {rd }}$ tertile: $\left.3.8 \% ; \mathrm{P}<0.01\right)$. Conclusion: Atherosclerotic plaque density evaluated by 64-MDCT significantly associates with plaque composition assessed by IVUS-VH.

DESCRIPTORS: Tomography, spiral computed. Ultrasonography, interventional. Coronary artery disease/pathology.

padrão coronário multiarterial ou síndrome coronária aguda recente $(<2$ semanas). Constituíram-se critérios de exclusão do estudo: fibrilação atrial, contraindicação a betabloqueador, insuficiência renal crônica (creatinina $>2 \mathrm{mg} \%$ ) e disfunção ventricular grave. A idade do paciente não foi um limitante para a entrada no estudo. A TCMD foi realizada em um intervalo inferior a 72 horas, antes do procedimento intervencionista. As imagens de HV foram adquiridas de todos os pacientes por ocasião da intervenção percutânea. Em cada paciente foram analisados pelo menos $40 \mathrm{~mm}$ de comprimento de um vaso epicárdico principal livre de stent ou tratamento cirúrgico prévio, sendo, sempre que possível, analisados os três vasos epicárdicos principais. O protocolo de estudo foi aprovado pelo comitê de ética local. O consentimento livre e esclarecido foi fornecido pelos participantes quando de sua inclusão no estudo.

\section{Tomografia computadorizada com múltiplos detectores: protocolo de aquisição e análise das imagens}

Todas as TCMDs foram realizadas em tomógrafo de 64 colunas de detectores Aquillion 64 (Toshiba America Medical Systems, Tustin, Estados Unidos), utilizando protocolo de aquisição de imagem semeIhante ao empregado em outros estudos com participação de nossa instituição ${ }^{11}$. Para a realização do exame, os pacientes receberam nitrato sublingual; nos casos em que a frequência cardíaca era > 65 bpm, os pacientes receberam metoprolol endovenoso até a dose máxima de $15 \mathrm{mg}$. Inicialmente obtinha-se o escore de cálcio por meio da aquisição prospectiva sincronizada pelo eletrocardiograma, usando colimação de $4 \mathrm{~mm}$ por $3 \mathrm{~mm}$. Independentemente do valor do escore de cálcio, obteve-se uma fase contrastada de todos os pacientes. Para a angiotomografia, o protocolo de aquisição era modificado, utilizando-se colimação de $64 \mathrm{~mm}$ por $0,5 \mathrm{~mm}$ e tempo de rotação mínimo (400 ms). Os demais parâmetros de aquisi- 
Falcão JLAA, et al. Associação Entre a Densidade Radiológica da Placa à Tomografia de Coronárias com 64 Colunas de Detectores e a Composição da Placa ao Ultrassom Intravascular com Técnica de Histologia Virtual: Resultados de uma Comparação Pareada Prospectiva. Rev Bras Cardiol Invas. 2009;17(3):327-34.

ção eram ajustados conforme sexo, peso e frequência cardíaca do paciente. Após a injeção de contraste com lopamidol 370 mg iodo/ml (Schering, Berlim, Alemanha), a aquisição helicoidal das imagens era iniciada quando se alcançava atenuação luminar de 180 unidades Hounsfield (UH) na aorta torácica descendente. Uma vez concluída a aquisição, realizavase a reconstrução tridimensional das imagens em diferentes fases do ciclo cardíaco e os dados eram transferidos para uma estação de trabalho específica (Vítrea 2 v3.9, Vital Images Inc., Plymouth, Estados Unidos). Para a análise quantitativa das artérias coronárias foram utilizados os programas Coronary Vessel Probe e SUREPlaque (Vital Images Inc., Plymouth, Estados Unidos). Todas as análises foram realizadas por operadores que desconheciam os dados clínicos, angiográficos e ultrassonográficos dos pacientes, seguindo um plano de análise preestabelecido. Primeiramente, avaliava-se a atenuação luminar média na aorta ascendente - parâmetro utilizado para auxiliar a definir o limiar para detecção do contorno luminar dos vasos. Em seguida, realizava-se o traçado dos contornos da luz e do vaso de todos os segmentos de forma semiautomática, o que permitia delimitar a parede arterial (placa média). Para avaliar a composição da parede arterial, foi medida sua densidade radiológica média (em UH). A parede arterial foi também dividida em componentes de diferentes densidades radiológicas (componente de baixa densidade, componente de média densidade e componente calcificado). Os limiares desses componentes foram definidos individualmente para cada paciente. Para a definição do limiar do componente calcificado (alta densidade), foram utilizados pontos de calcificação previamente identificados ao escore de cálcio. Para a definição dos componentes de baixa densidade e de densidade intermediária, utilizou-se o limiar de 30-50 UH, conforme previamente empregado em outros estudos ${ }^{12}$. Áreas com densidade inferior a esse valor eram consideradas de baixa densidade, enquanto valores de atenuação maiores que 30-50 UH e inferiores aos definidos para o componente calcificado eram atribuídos a um componente de densidade intermediária (Figura 1). A contribuição porcentual de cada componente para a parede arterial, bem como a razão "área do componente de baixa densidade: área do componente calcificado", foi obtida para posterior análise.

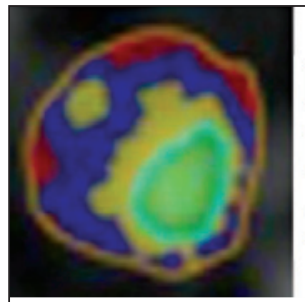

\section{$\square$ Baixa densidade}

$\square$ Densidade intermediária

\section{Cálcio (alta densidade)}

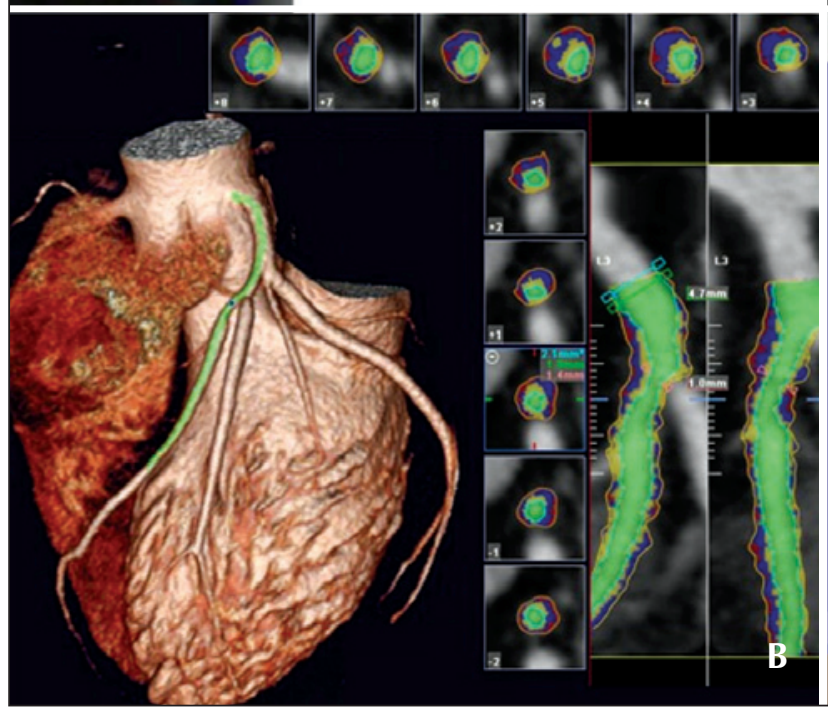

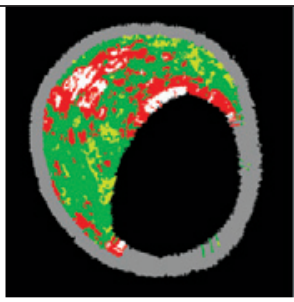

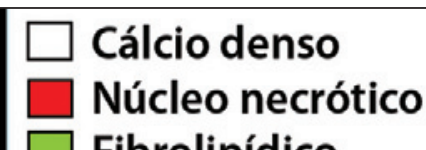

Fibrolipídico

Fibroso

Média (normal)

C

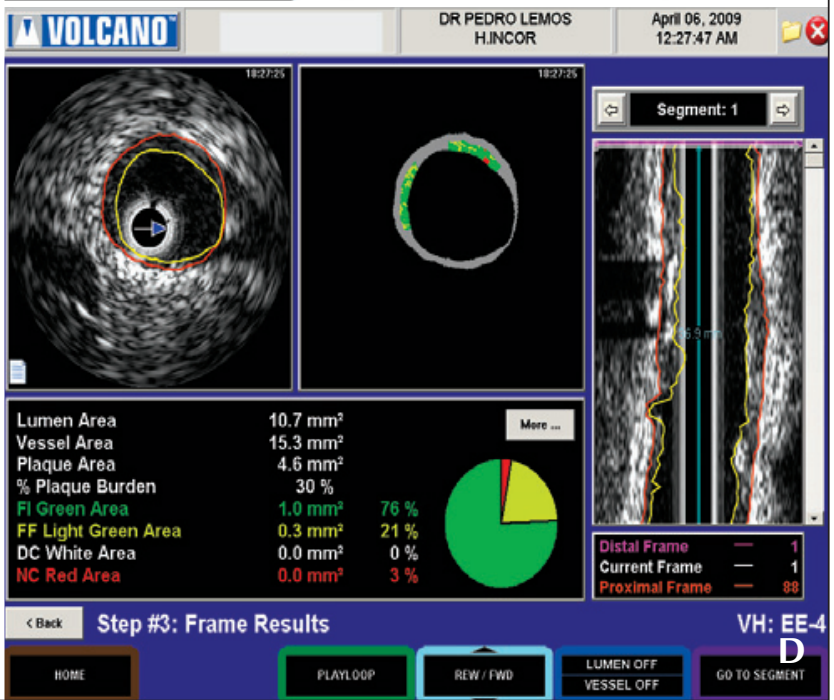

Figura 1 - Imagens da placa aterosclerótica coronária à tomografia computadorizada com múltiplos detectores (TCMD) em tomógrafo de 64 colunas de detectores e à histologia virtual (HV). Em A, corte seccional transversal à TCMD, demonstrando componentes de diferentes densidades radiológicas da parede arterial. Em B, coração na visão volumétrica à TCMD com emprego dos programas Vessel Probe e SUREPlaque para análise da composição da parede arterial. Em C, corte seccional transversal à HV, demonstrando componentes da placa aterosclerótica e média "normal". Em D, cortes seccionais transversais e longitudinal da artéria ao ultrassom intracoronário com técnica de HV com emprego do programa pcVH 2.2 para avaliar a composição da placa aterosclerótica. 
Falcão JLAA, et al. Associação Entre a Densidade Radiológica da Placa à Tomografia de Coronárias com 64 Colunas de Detectores e a Composição da Placa ao Ultrassom Intravascular com Técnica de Histologia Virtual: Resultados de uma Comparação Pareada Prospectiva. Rev Bras Cardiol Invas. 2009;17(3):327-34.

\section{Ultrassom intracoronário com técnica de histologia virtual: protocolo de aquisição e análise das imagens}

As imagens de HV foram obtidas por ocasião da intervenção percutânea. Utilizou-se um cateter eletrônico de múltiplos feixes de ultrassom com 2,9 F de diâmetro (Eagle Eye ${ }^{\circledR}$, Volcano Corporation Inc., Rancho Cordova, Estados Unidos), que foi introduzido no terço distal do vaso de interesse após a administração de nitrato intracoronário. O cateter foi conectado a um console apropriado (InVision Gold ${ }^{\circledR}$, Volcano Corporation Inc., Rancho Cordova, Estados Unidos), capaz de gerar imagens de ultrassom em escala de cinza e no modo HV simultaneamente. As imagens foram gravadas durante a retirada automática do cateter a uma velocidade constante de $0,5 \mathrm{~mm} / \mathrm{s}$ (dispositivo motorizado R-100 ${ }^{\circledR}$, Volcano Corporation Inc., Rancho Cordova, Estados Unidos). As imagens de HV foram obtidas a uma taxa de aquisição de 1 quadro/batimento cardíaco (no pico da onda "R"). A análise das imagens foi realizada por um operador que desconhecia as características clínicas do paciente, utilizando-se programa específico (pcVH 2.2 ${ }^{\circledR}$, Volcano Corporation Inc., Rancho Cordova, Estados Unidos) para a delimitação dos contornos vasculares e para a avaliação da composição da parede vascular e de suas dimensões. O programa funciona da seguinte forma: inicialmente os limites da luz e da lâmina elástica externa são traçados de forma semiautomática para cada quadro (imagem em escala de cinza); depois de definidos os limites da parede arterial (placa média), o programa realiza as medidas de área e a análise dos componentes da placa, identificando quatro componentes da placa aterosclerótica propriamente dita (fibroso, fibrolipídico, núcleo necrótico e cálcio denso) e a média "normal" (Figura 1). A contribuição porcentual de cada componente da placa (excluindo-se a média) e a razão necrose:cálcio (área do componente núcleo necrótico:área do componente cálcio denso) foram calculadas.

\section{Comparação das imagens entre TCMD e HV}

As imagens de HV e de TCMD foram pareadas utilizando pontos de referência anatômicos facilmente identificáveis nos vasos aos dois métodos (óstio, ramos laterais, entre outros). As distâncias das referências ao óstio do vaso foram anotadas. Essas anotações possibilitaram parear os segmentos, de forma a comparar as mesmas regiões aos dois métodos. Para fins de comparação, cada vaso (exceto o tronco da artéria coronária esquerda) foi dividido em subsegmentos de $4 \mathrm{~mm}$ (unidades básicas para a análise). Em razão de sua menor extensão, e com o objetivo de facilitar a comparação, o tronco da coronária esquerda foi computado como um segmento único.

\section{Análise estatística}

Variáveis categóricas são apresentadas como valor absoluto e porcentagem, variáveis contínuas nor- mais são apresentadas como média e desvio padrão, e variáveis contínuas que não seguem a distribuição normal são apresentadas como mediana e intervalo interquartil. As variáveis contínuas que não seguem a distribuição normal foram analisadas para correlação com o teste não-paramétrico de Spearman e comparadas por meio do teste de múltiplas amostras independentes de Kruskal-Wallis. $\mathrm{O}$ valor de $\mathrm{P}<0,05$ foi considerado significativo. Utilizou-se o programa estatístico SPSS (versão 13.0) para a realização das análises.

\section{RESULTADOS}

A população de pacientes estudada $(n=21)$ apresentou prevalência de diabetes melito de 48\%, 38\% eram multiarteriais, 38\% relatavam infarto do miocárdio prévio e quase a metade dos pacientes foi admitida com diagnóstico de síndrome coronária aguda (Tabela 1).

No total, 70 vasos foram analisados (média de 3,3 vasos/paciente), compreendendo 5.972 quadros de ultrassom (HV) e 5.233 secções transversais de TCMD. Os vasos avaliados foram divididos em 641 subsegmentos (633 subsegmentos de $4 \mathrm{~mm}$ e 8 troncos de artéria coronária esquerda), que constituíram as unidades básicas de comparação do estudo. A Tabela 2 apresenta os resultados da análise da composição da placa do paciente, do vaso e dos subsegmentos à TCMD e à HV - observando-se que os resultados se assemelham em qualquer nível de análise. Em vista do exposto e procurando atingir um critério mais minucioso de análise, os dados a seguir referem-se aos subsegmentos. A área da parede arterial na secção transversal à TCMD se correlacionou com a área da placa média à HV (coeficiente de correlação de Spearman de 0,55; $\mathrm{P}<0,01)$. Observou-se, entretanto, uma superestimativa da área da parede arterial à TCMD

TABELA 1

Características dos pacientes $(n=21)$

\begin{tabular}{|c|c|}
\hline Características & \\
\hline Idade em anos, média \pm desvio padrão & $57 \pm 10$ \\
\hline Sexo masculino, n (\%) & $15(71)$ \\
\hline Diabetes melito, n (\%) & $10(48)$ \\
\hline Hipertensão arterial sistêmica, n (\%) & $15(71)$ \\
\hline Hipercolesterolemia, n (\%) & $12(57)$ \\
\hline Tabagismo atual, n (\%) & $7(33)$ \\
\hline Infarto prévio, n (\%) & $8(38)$ \\
\hline Doença multiarterial, n (\%) & $8(38)$ \\
\hline Síndrome coronária aguda à admissão, n (\%) & $10(48)$ \\
\hline $\begin{array}{l}\text { Escore total de cálcio - Agatston, } \\
\text { mediana (percentis: 5-95) }\end{array}$ & $19(0-698)$ \\
\hline Pacientes com escore de cálcio > 400, n (\%) & $2(10)$ \\
\hline
\end{tabular}


Falcão JLAA, et al. Associação Entre a Densidade Radiológica da Placa à Tomografia de Coronárias com 64 Colunas de Detectores e a Composição da Placa ao Ultrassom Intravascular com Técnica de Histologia Virtual: Resultados de uma Comparação Pareada Prospectiva. Rev Bras Cardiol Invas. 2009;17(3):327-34.

TABELA 2

Características da parede arterial avaliada ao ultrassom com técnica de histologia virtual e à tomografia computadorizada com 64 colunas de detectores segundo o nível de análise: paciente, vaso e subsegmento

\begin{tabular}{|c|c|c|c|}
\hline & $\begin{array}{l}\text { Nível paciente } \\
\text { (21 pacientes) }\end{array}$ & $\begin{array}{l}\text { Nível vaso } \\
\text { (70 vasos) }\end{array}$ & $\begin{array}{l}\text { Nível subsegmento } \\
\text { (641 subsegmentos) }\end{array}$ \\
\hline \multicolumn{4}{|l|}{ Histologia virtual } \\
\hline Área da placa média, mm² & $5,6(3-10,4)$ & $6,1(2,8-11,4)$ & $5,3(1,7-11,9)$ \\
\hline Área da média, mm² & $3,3(2,4-4,4)$ & $3,4(2,3-5)$ & $3,2(1,6-4,7)$ \\
\hline Área da placa, mm² & $2,7(0,6-6,6)$ & $2,4(0,2-6,9)$ & $1,7(0,0-8,1)$ \\
\hline Componente fibroso, \% & $65,1(56,2-73,1)$ & $64,9(51,6-74)$ & $65,3(45,2-78,3)$ \\
\hline Componente fibrolipídico, \% & $16,1(8-29,1)$ & $18,5(7,8-37,9)$ & $18,1(45,2-78,3)$ \\
\hline Componente núcleonecrótico, \% & $12,3(5,4-21,4)$ & $10,4(1,4-23,4)$ & $9,0(0-27,4)$ \\
\hline Componente cálcio denso, \% & $4,4(1,5-12,6)$ & $3,4(0,2-14,1)$ & $2,6(0-18)$ \\
\hline Razão necrose:cálcio & $2,7(0,9-5,4)$ & $2,9(0,9-12,5)$ & $2,9(0,74-16)$ \\
\hline \multicolumn{4}{|l|}{ TCMD-64 } \\
\hline Área da parede arterial, mm² & $9,7(6,1-17,8)$ & $9,5(5,7-18,5)$ & $8,9(4,6-16,4)$ \\
\hline Densidade global daparede, UH & $154,5(113-278,1)$ & $159,3(112,7-258,3)$ & $147,6(103-267,3)$ \\
\hline Componente de baixa densidade, \% & $4,3(1,1-14,4)$ & $4,1(0,1-16,9)$ & $2,9(0,03-18,1)$ \\
\hline Atenuação mediana, UH & $17,3(-3-33,6)$ & $17,2(2,7-34,5)$ & $18,5(-0,8-35,2)$ \\
\hline Componente de densidade intermediária, \% & $91,1(64,8-95,9)$ & $88,9(49,4-97,8)$ & $92,7(53,3-98,9)$ \\
\hline Atenuação mediana, UH & $148,4(106,9-266,2)$ & $148,4(92,6-246)$ & $146,9(92,9-241,7)$ \\
\hline Componente calcificado, \% & $4,2(0,1-32,7)$ & $4,1(0,0-41,3)$ & $1,1(0-36,2)$ \\
\hline Atenuação mediana, UH & $369,5(204,6-611,5)$ & $356,2(196,4-613,6)$ & $351,5(182,1-601,6)$ \\
\hline $\begin{array}{l}\text { Razão componente de baixa densidade: } \\
\text { componente calcificado }\end{array}$ & $1,45(0,07-27,1)$ & $0,93(0,08-100,6)$ & $0,76(0,02-45,45)$ \\
\hline
\end{tabular}

Números expressos como medianas (percentis 5 e 95).

OBS: Não houve diferenças significativas entre os diferentes níveis de análise: por paciente, por vaso ou por subsegmento.

TCMD-64 = tomografia computadorizada com múltiplos detectores em tomógrafo de 64 colunas de detectores.

comparativamente à HV (mediana de $3,2 \mathrm{~mm}^{2}$ ). Ao analisarmos à HV o conjunto placa-média, o componente predominante em nossa amostra foi o de média normal, que respondeu por $65 \%$ da área da parede do vaso. Ao considerarmos apenas a placa aterosclerótica, o componente fibroso foi o mais frequente, seguido pelos componentes fibrolipídico, núcleo necrótico e cálcio denso. A quantidade do componente núcleo necrótico foi cerca de três vezes maior que a do componente calcificado. A mediana de atenuação da parede arterial à TCMD foi de 150 UH. O componente de densidade intermediária foi o mais frequente, respondendo por cerca de $90 \%$ da placa. Os demais componentes responderam por menos de $5 \%$ da parede arterial. A quantidade de componente de baixa densidade foi cerca de $24 \%$ menor que a do componente calcificado. Considerando que a média normal foi o componente da parede arterial preponderante neste estudo, verificamos que cerca de 10\% ( $n=64)$ dos subsegmentos analisados não apresentavam placa aterosclerótica à $\mathrm{HV}$, ou seja, possuíam apenas média (segmentos "sadios"). À TCMD, esses subsegmentos apresentavam densidade mediana da parede arterial de $138 \mathrm{UH}$, sendo observado que o componente de densidade intermediária respondeu por 93,8\% da composição da parede nesta amostra - o que sugere que o componente média da HV seja identificado predominantemente como um componente de densidade intermediária à TCMD.

Houve correlação significativa entre os componentes da placa aterosclerótica à HV e os componentes de diferentes densidades da parede arterial à TCMD individualmente (coeficientes de correlação de Spearman $<0,15)$. De modo a avaliar melhor a associação entre a densidade global da parede arterial à TCMD e a composição da placa aterosclerótica à HV, os subsegmentos foram divididos em três grupos de mesmo tamanho (tercis), de acordo com a densidade global da parede arterial à TCMD: 1) parede arterial com baixa densidade (1ำ tercil): de 43,8 a 128,1 UH (mediana: $113,3 \cup H)$; 2) parede arterial com densidade intermediária ( ${ }^{\circ}$ tercil): de 128,2 a 177,7 HU (mediana: $147,4 \cup H)$; e 3) parede arterial com alta densidade ( $3^{\circ}$ tercil): de 177,8 a 439,5 HU (mediana: 221,3 UH). Os três grupos de subsegmentos de diferentes tercis de densidade radiológica à TCMD apresentaram composição significativamente diferente à HV. Nesse sen- 
Falcão JLAA, et al. Associação Entre a Densidade Radiológica da Placa à Tomografia de Coronárias com 64 Colunas de Detectores e a Composição da Placa ao Ultrassom Intravascular com Técnica de Histologia Virtual: Resultados de uma Comparação Pareada Prospectiva. Rev Bras Cardiol Invas. 2009;17(3):327-34.

tido, o aumento da densidade radiológica da parede arterial à TCMD associou-se a aumento da contribuição porcentual dos componentes cálcio denso e núcleo necrótico e a redução da contribuição porcentual do componente fibrolipídico à HV, bem como a menor razão necrose:cálcio (Figura 2).

\section{DISCUSSÃO}

O principal achado deste estudo foi que a composição da parede arterial (em termos de densidade radiológica global) à TCMD com tomógrafo de 64 colunas de detectores foi significativamente associada com a composição da placa aterosclerótica à HV.

A análise da correlação dos componentes da parede arterial à TCMD (conforme diferentes densidades radiológicas) apresentou fraca correspondência com os componentes da placa aterosclerótica à HV, achado corroborado por outros pesquisadores ${ }^{13-15}$. Isso pode ser atribuído, entre outros fatores, à capacidade da TCMD em distinguir três componentes da parede arterial de diferentes densidades radiológicas, enquanto à HV a parede é dividida em cinco constituintes (quatro componentes da placa e a média normal). Não há, portanto, como estabelecer correlação direta compo- nente a componente entre os dois métodos (correlação um a um). Além do exposto, existe considerável superposição nos limites de densidade radiológica dos componentes não calcificados à TCMD. Outro ponto a se considerar é a menor resolução tanto espacial como temporal da TCMD em relação à $H V$, que pode não identificar áreas de microcalcificações no interior de núcleos lipídicos, as quais ocasionalmente poderiam ser caracterizadas como regiões de densidade intermediária ${ }^{15,16}$.

Embora a correlação entre os componentes individuais da placa aos dois métodos seja fraca, considerando a densidade global da parede arterial, a TCMD mostrou-se sensível a variações na contribuição porcentual dos constituintes da placa à HV, excetuandose o componente fibroso. Talvez isso possa ser explicado por este ser o constituinte predominante da placa aterosclerótica em nossa amostra. Desse modo, placas com baixa densidade radiológica à TCMD apresentaram maior conteúdo fibrolipídico à HV. Placas com alta densidade radiológica apresentaram maior conteúdo de cálcio denso e núcleo necrótico, achado também sugerido por outros autores em estudo recente sobre esse tema ${ }^{15}$. A aparente contradição entre a associação de componente núcleo necrótico (com alto

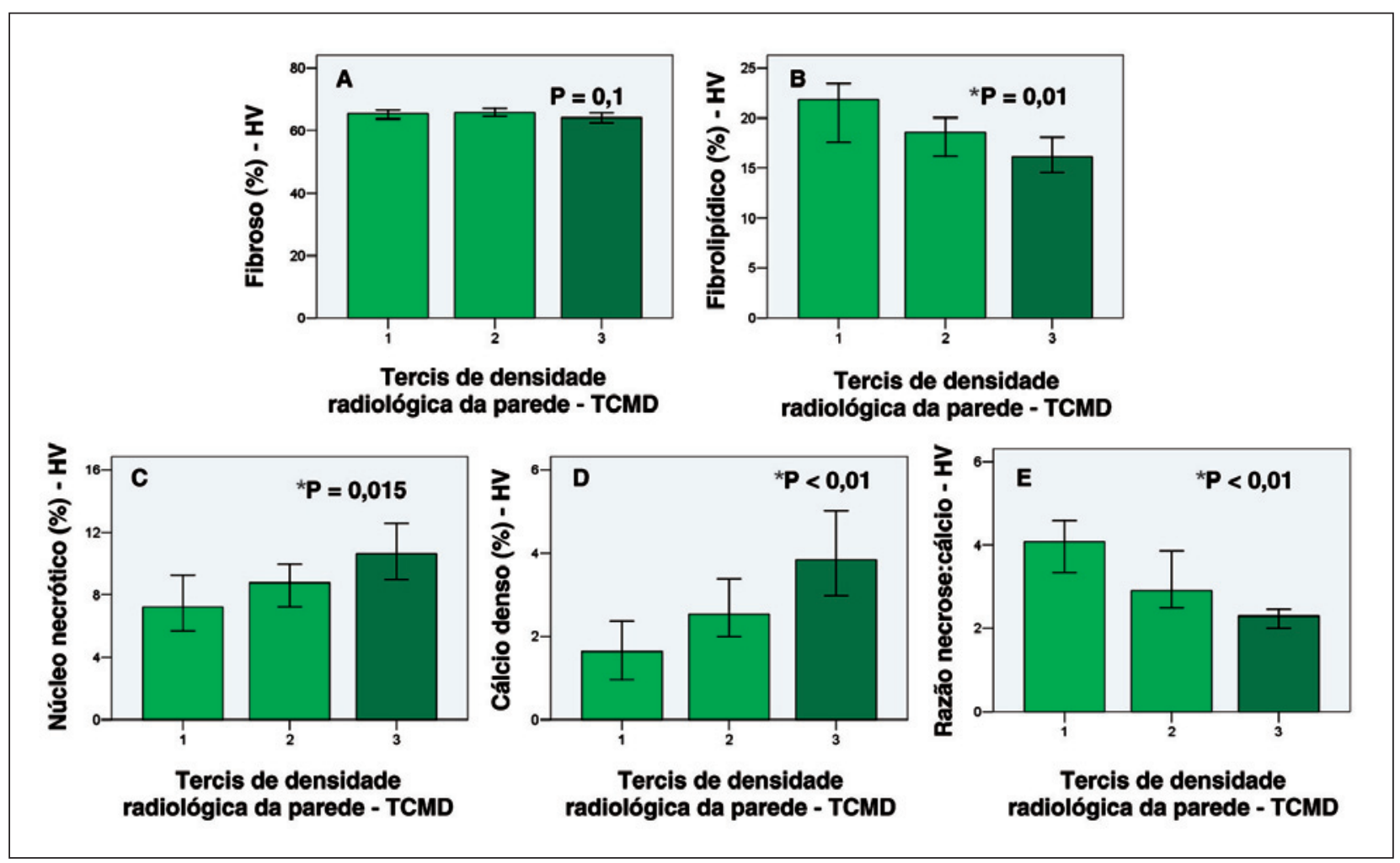

Figura 2 - Comparação entre a composição da parede arterial à tomografia computadorizada com múltiplos detectores (TCMD) em tomógrafo de 64 colunas de detectores e a composição da placa à histologia virtual (HV). Os subsegmentos foram divididos em três grupos (tercis), de acordo com sua densidade radiológica global à TCMD, e comparados com a mediana da contribuição porcentual dos diferentes componentes da placa aterosclerótica à HV: fibroso (A), fibrolipídico (B), núcleo necrótico (C), cálcio denso (D) e razão necrose:cálcio (E). As barras de erros representam os intervalos de confiança de $95 \%$. * Estatisticamente significante. 
Falcão JLAA, et al. Associação Entre a Densidade Radiológica da Placa à Tomografia de Coronárias com 64 Colunas de Detectores e a Composição da Placa ao Ultrassom Intravascular com Técnica de Histologia Virtual: Resultados de uma Comparação Pareada Prospectiva. Rev Bras Cardiol Invas. 2009;17(3):327-34.

conteúdo lipídico) e alta densidade radiológica à TCMD pode ser explicada pela forte correlação existente entre os componentes cálcio denso e núcleo necrótico à HV (coeficiente de correlação de Spearman $=0,75$; $P<0,01)$, achado já relatado por outros investigadores ${ }^{17,18}$. Como existe incremento do conteúdo núcleo necrótico quando se aumenta a contribuição do componente cálcio denso, essa análise precisa ser esmiuçada com o auxílio da razão necrose:cálcio. Quando se analisa essa razão, observa-se que quanto mais densa a parede arterial à TCMD menor a razão necrose:cálcio à $\mathrm{HV}$, o que sugere que o componente núcleo necrótico deve corresponder a um componente de baixa densidade radiológica à TCMD. Nossos achados diferiram parcialmente dos de Choi et al. ${ }^{16}$, que encontraram correlação positiva entre a densidade global de placas não calcificadas à TCMD e o conteúdo relativo de componente fibroso, e correlação inversa quanto ao constituinte núcleo necrótico, fato possivelmente explicado por diferença de amostra entre os estudos.

Como achado secundário do estudo, verificou-se que, em termos quantitativos, as medidas da área seccional da parede arterial à TCMD e à HV se correlacionaram moderadamente, observando-se superestimativa das dimensões da área da parede arterial à TCMD. Esse achado, embora não consensual à literatura, já havia sido relatado por outros autores ${ }^{8,19,20}$, podendo ser parcialmente atribuído a artefatos radiológicos tipo blooming (durante a aquisição da imagem radiológica, o deslocamento rápido de ponto s com alta densidade radiológica pode afetar a mensuração da densidade das regiões adjacentes, aumentando a densidade dos pixels das regiões circunvizinhas, o que leva à superestimação da área mensurada de regiões de alta densidade radiológica), que aumentam a área de pontos intensamente calcificados na TCMD, e ao fato de os limites externos da parede arterial serem diferentes nos dois métodos avaliados (na HV, a lâmina elástica externa separa a média da adventícia, enquanto na TCMD o limite externo do vaso separa a adventícia da gordura periadventicial).

Nosso relato deve ser visto como um estudo piloto, cujos achados necessitam de confirmação posterior. Um pequeno número de pacientes foi analisado, o que obviamente limita extrapolações dos achados para grandes populações. O grau de calcificação das artérias coronárias, os parâmetros da qualidade da imagem radiológica (atenuação luminal, relação sinalruído e ruído de atenuação) e o protocolo de aquisição das imagens radiológicas empregados podem interferir com os achados do estudo ${ }^{21,22}$.

O papel da HV como ferramenta de pesquisa clínica para a avaliação da composição da placa aterosclerótica está em fase de validação, de forma que ainda não podemos considerar o método padrão de referência em um estudo de comparação entre duas tecnologias recentes. Entretanto, a HV tem sido utilizada em estudos clínicos para acompanhar modificações nas dimensões e na composição da placa aterosclerótica $^{23}$. A despeito das limitações citadas, nosso estudo sugere que a TCMD é um método candidato a ser empregado para avaliar variações da constituição da placa aterosclerótica de forma não-invasiva em estudos de evolução da doença arterial coronária e em estudos de progressão/regressão de placas ateroscleróticas coronárias.

\section{CONCLUSÃO}

Nosso estudo demonstra que a densidade radiológica da parede arterial coronária, em imagens obtidas com tomógrafos computadorizados com 64 colunas de detectores, se associa com a composição da placa aterosclerótica ao ultrassom intracoronário com técnica de HV. Esses achados sugerem que a tomografia computadorizada pode ser uma ferramenta útil para a caracterização não-invasiva da placa aterosclerótica coronária.

\section{CONFLITO DE INTERESSES}

Os autores declararam inexistência de conflito de interesses relacionados a este artigo.

\section{REFERÊNCIAS BIBLIOGRÁFICAS}

1. Becker CR, Nikolaou K, Muders M, Babaryka G, Crispin A, Schoepf UJ, et al. Ex vivo coronary atherosclerotic plaque characterization with multi-detector-row CT. Eur Radiol. 2003;13(9):2094-8.

2. Agatston AS, Janowitz WR, Hildner FJ, Zusmer NR, Viamonte M Jr, Detrano R. Quantification of coronary artery calcium using ultrafast computed tomography. J Am Coll Cardiol. 1990;15(4):827-32.

3. Nishimura RA, Edwards WD, Warnes CA, Reeder GS, Holmes DR Jr, Tajik AJ, et al. Intravascular ultrasound imaging: in vitro validation and pathologic correlation. J Am Coll Cardiol. 1990;16(1):145-54.

4. Di Mario C, The SH, Madretsma S, van Suylen RJ, Wilson RA, Bom N, et al. Detection and characterization of vascular lesions by intravascular ultrasound: an in vitro study correlated with histology. J Am Soc Echocardiogr. 1992;5(2):135-46.

5. Nair A, Kuban BD, Tuzcu EM, Schoenhagen P, Nissen SE, Vince DG. Coronary plaque classification with intravascular ultrasound radiofrequency data analysis. Circulation. 2002; 106(17):2200-6.

6. Nasu K, Tsuchikane E, Katoh O, Vince DG, Virmani R, Surmely JF, et al. Accuracy of in vivo coronary plaque morphology assessment: a validation study of in vivo virtual histology compared with in vitro histopathology. J Am Coll Cardiol. 2006;47(12):2405-12.

7. Kopp AF, Schroeder S, Baumbach A, Kuettner A, Georg C, Ohnesorge B, et al. Non-invasive characterization of coronary lesion morphology and composition by multislice CT: first results in comparison with intracoronary ultrasound. Eur Radiol. 2001;11(9):1607-11.

8. Leber AW, Becker A, Knez A, von Ziegler F, Sirol M, Nikolaou $K$, et al. Accuracy of 64-slice computed tomography to classify and quantify plaque volumes in the proximal coronary system: a comparative study using intravascular ultrasound. J Am Coll Cardiol. 2006;47(3):672-7 
Falcão JLAA, et al. Associação Entre a Densidade Radiológica da Placa à Tomografia de Coronárias com 64 Colunas de Detectores e a Composição da Placa ao Ultrassom Intravascular com Técnica de Histologia Virtual: Resultados de uma Comparação Pareada Prospectiva. Rev Bras Cardiol Invas. 2009;17(3):327-34.

9. Leber AW, Knez A, Becker A, Becker C, von Ziegler F, Nikolaou K, et al. Accuracy of multidetector spiral computed tomography in identifying and differentiating the composition of coronary atherosclerotic plaques: a comparative study with intracoronary ultrasound. J Am Coll Cardiol. 2004;43(7): 1241-7.

10. Achenbach S, Moselewski F, Ropers D, Ferencik M, Hoffmann $U$, MacNeill B, et al. Detection of calcified and noncalcified coronary atherosclerotic plaque by contrast-enhanced, submillimeter multidetector spiral computed tomography: a segmentbased comparison with intravascular ultrasound. Circulation. 2004;109(1):14-7.

11. Miller JM, Rochitte CE, Dewey M, Arbab-Zadeh A, Niinuma $\mathrm{H}$, Gottlieb I, et al. Diagnostic performance of coronary angiography by 64-row CT. N Engl J Med. 2008;359(22):2324-36.

12. Cordeiro MA, Lima JA. Atherosclerotic plaque characterization by multidetector row computed tomography angiography. J Am Coll Cardiol. 2006;47(8 Suppl):C40-7.

13. Brodoefel H, Burgstahler C, Heuschmid M, Reimann A, Khosa F, Kopp A, et al. Accuracy of dual-source CT in the characterization of non-calcified plaque: use of a colorcoded analysis compared with virtual histology intravascular ultrasound. Br J Radiol. 2009;82(982):805-12.

14. Brodoefel H, Burgstahler C, Sabir A, Yam CS, Khosa F, Claussen $\mathrm{CD}$, et al. Coronary plaque quantification by voxel analysis: dual-source MDCT angiography versus intravascular sonography. AJR Am J Roentgenol. 2009;192(3):W84-9.

15. Pundziute G, Schuijf JD, Jukema JW, Decramer I, Sarno G, Vanhoenacker PK, et al. Head-to-head comparison of coronary plaque evaluation between multislice computed tomography and intravascular ultrasound radiofrequency data analysis. JACC Cardiovasc Interv. 2008;1(2):176-82.

16. Choi BJ, Kang DK, Tahk SJ, Choi SY, Yoon MH, Lim HS, et al. Comparison of 64-slice multidetector computed tomography with spectral analysis of intravascular ultrasound backscatter signals for characterizations of noncalcified coronary arterial plaques. Am J Cardiol. 2008;102(8):988-93.

17. Missel E, Mintz GS, Carlier SG, Qian J, Shan S, Castellanos $C$, et al. In vivo virtual histology intravascular ultrasound correlates of risk factors for sudden coronary death in men: results from the prospective, multi-centre virtual histology intravascular ultrasound registry. Eur Heart J. 2008;29(17): 2141-7.

18. Murray SW, Palmer ND. What is behind the calcium? The relationship between calcium and necrotic core on virtual histology analyses. Eur Heart J. 2009;30(1):125-6.

19. Kitagawa T, Yamamoto $H$, Ohhashi N, Okimoto $T$, Horiguchi J, Hirai $\mathrm{N}$, et al. Comprehensive evaluation of noncalcified coronary plaque characteristics detected using 64-slice computed tomography in patients with proven or suspected coronary artery disease. Am Heart J. 2007;154(6):1191-8.

20. Sun J, Zhang Z, Lu B, Yu W, Yang Y, Zhou Y, et al. Identification and quantification of coronary atherosclerotic plaques: a comparison of $64-\mathrm{MDCT}$ and intravascular ultrasound. AJR Am J Roentgenol. 2008;190(3):748-54.

21. Cademartiri F, Mollet NR, Runza G, Bruining N, Hamers R, Somers $\mathrm{P}$, et al. Influence of intracoronary attenuation on coronary plaque measurements using multislice computed tomography: observations in an ex vivo model of coronary computed tomography angiography. Eur Radiol. 2005;15(7): 1426-31.

22. Cademartiri F, La Grutta L, Runza G, Palumbo A, Maffei E, Mollet NR, et al. Influence of convolution filtering on coronary plaque attenuation values: observations in an ex vivo model of multislice computed tomography coronary angiography. Eur Radiol. 2007;17(7):1842-9.

23. Hong MK, Park DW, Lee CW, Lee SW, Kim YH, Kang DH, et al. Effects of statin treatments on coronary plaques assessed by volumetric virtual histology intravascular ultrasound analysis. JACC Cardiovasc Interv. 2009;2(7):679-88. 